\title{
A CONVERGENCE TEST FOR WALSH-FOURIER SERIES
}

\author{
SHIGEKI YANO
}

(Received September 16,1954)

The purpose of this note is to show that the convergence test of Hardy and Littlewood [2] or [5] for trigonometric Fourier series is also valid for Walsh-Fourier series.

Let $f(x)$ be a periodic function with period 1 and integrable in the interval $(0,1)$, and its Walsh-Fourier series be

$$
f(x) \sim \sum_{n=0}^{\infty} c_{n} \psi_{n}(x)
$$

where the definition of the Walsh functions is used in the sense due to Paley [3] or Fine [1]. For the notations and fundamental results on the Walsh functions, the reader is refered to the paper of Fine [1].

THEOREM. The Walsh-Fourier series (1) of $f(x)$ converges at the point $x_{0}$ to the value $f\left(x_{0}\right)$, if the following two conditions are satisfied:

$$
\begin{gathered}
\int_{0}^{h}\left|f\left(x_{0}+t\right)-f\left(x_{0}\right)\right| d t=o\left(|h| / \log \frac{1}{|h|}\right), \quad h \rightarrow 0 ; \\
c_{n}=O\left(n^{-\delta}\right), \quad \delta>0 .
\end{gathered}
$$

Proof. It is easy to see that the condition (i) implies

$$
\int_{0}^{h}\left|f\left(x_{0}+t\right)-f\left(x_{0}\right)\right| d t=o\left(h / \log \frac{1}{h}\right), \quad h \rightarrow+0
$$

(see [4; proof of Lemma 11]). Now, if we set

$$
g(x)=f\left(x_{0}+x\right)-f\left(x_{0}\right),
$$

the Walsh-Fourier series of $g(x)$ is (see Fine [1; Theorem II])

$$
c_{0}-f\left(x_{0}\right)+\sum_{n=1}^{\infty} c_{n} \psi_{n}\left(x_{0}\right) \psi_{n}(x)
$$

and $g(x)$ satisfies the condition (2) for $x=0$ instead of $x=x_{0}$, and the order condition (ii) for the coefficients. Since, instead of $f(x)$ we may consider $g(x)$, let us assume that $x_{0}=0, f\left(x_{0}\right)=0$,

$$
\begin{aligned}
\int_{0}^{h}|f(t)| d t & =o\left(h / \log \frac{1}{h}\right), \quad h \rightarrow+0 \\
c_{n} & =O\left(n^{-\delta}\right), \quad 0<\delta<1
\end{aligned}
$$

and under these conditions we shall prove that the series $\sum_{n=0}^{\infty} c_{n}$ converges to. 
the value 0 .

Let $s_{n}(x)$ be the $n$-th partial sum of the series (1):

$$
s_{n}(x)=\sum_{k=0}^{n-1} c_{k} \psi_{k}(x)
$$

Then we have to prove that $s_{n}(0)$ converges to 0 . As is well known, $s_{2 n}(0)$ $=2^{n} \int_{0}^{2^{-n}} f(t) d t$, so that the condition ( $\left.\mathrm{i}^{\prime}\right)$ implies that $s_{2 n}(0)=o(1)$. Thus it is sufficient to prove that for $k=2^{n}+k^{\prime}, 0 \leqq k^{\prime}<2^{n}$

$$
s_{2 n+k^{\prime}}(0)-s_{2 n}(0)=\int_{0}^{1} \psi_{2 n}(t) D_{v^{\prime}}(t) f(t) d t
$$

converges to 0 as $n \rightarrow \infty$, where $D_{n}(t)$ is the Dirichlet kernel for the Walsh functions :

$$
D_{n}(t)=\sum_{k=0}^{n-1} \psi_{k}(t)
$$

Let $r=\frac{\delta}{2}$. We have

$$
\begin{aligned}
\int_{0}^{1} \psi_{2 n}(t) D_{k^{\prime}}(t) f(t) d t & =\left\{\int_{0}^{2^{-n}}+\int_{2^{-n}}^{2^{-[r n]}}+\int_{2-[r n]}^{1}\right\} \psi_{2 n}(t) D_{k k^{\prime}}(t) f(t) d t \\
& =P+Q+R .
\end{aligned}
$$

Since $\left|D_{l_{0}}(t)\right| \leqq k^{\prime}$, it follows by $\left(\mathrm{i}^{\prime}\right)$

(5) $\quad|P| \leqq \int_{0}^{2^{-n}}|f(t)|\left|D_{k^{\prime}}(t)\right| d t \leqq k^{\prime} \int_{0}^{2^{-n}}|f(t)| d t=O\left(2^{-n} \cdot 2^{n} / \log 2^{n}\right)=o$ (1).

Remenbering the inequality $\left|D_{k^{\prime}}(t)\right|<\frac{2}{t} \quad(0<t<1)$ (Fine 11 ; Lemma I in $\S 6]$ ), we have by integration by parts

$$
\begin{aligned}
|Q| & \leqq \int_{2^{-n}}^{2^{-[r n]}}|f(t)|\left|D_{k^{\prime}}(t)\right| d t \leqq 2 \int_{2^{-n}}^{2^{-[r n]}} \frac{|f(t)|}{t} d t \\
& =o(1)+o\left(\int_{2^{-n}}^{\left.2^{-i} \cdot n\right]} \frac{d t}{t \log \frac{1}{t}}\right) \\
& =o(1)+o\left(\log \log 2^{n}-\log \log 2^{[r n]}\right)=o(1),
\end{aligned}
$$

and it remains only to show $R=o(1)$. For the estimation of $R$, we use the following

Lemma. If $\chi(x) \sim \sum_{n=0}^{\infty} a_{n} \psi_{n}(x)$ is integrable and $h(x) \sim \sum_{n=0}^{\infty} b_{n} \psi_{n}(x)$ is of 
bounded variation in the interval $(0,1)$, then

$$
\int_{0}^{1} \chi(x) h(x) d x=\sum_{n=0}^{\infty} a_{n} b_{n}
$$

where the series on the right is convergent.

Taking for granted of this lemma, we have

$$
\begin{aligned}
R & =\sum_{j=0}^{\infty} c_{j} \int_{2^{-[r n]}}^{1} \psi_{j}(t) \psi_{2 n}(t) D_{k^{\prime}}(t) d t \\
& =\left\{\sum_{j=0}^{2^{n}-1}+\sum_{j=2^{n}}^{2^{n+1}-1}+\sum_{:^{n+1}}^{\infty}\right\} c_{j} \int_{2^{-[r n]}}^{1} \psi_{j}(t) \psi_{2 n}(t) D_{k^{\prime}}(t) d t \\
& =S+T+U .
\end{aligned}
$$

Since it is easily seen by the property of Walsh functions that

$$
\int_{2^{-l}}^{1} \psi_{2}^{m}(t) \psi_{j}(t) \psi_{j}(t) d t=0 \quad \text { for } 0 \leqq j, j^{\prime}<2^{m}, m \geqq l
$$

we get

(9)

$$
S=0
$$

and the same consideration gives

$$
U=0
$$

so that it remains only to show $T=o(1)$. Now

$$
\begin{aligned}
T & =\sum_{j=2^{n}}^{2^{n+1}-1} c_{j} \int_{2^{-[r n]}}^{1} \psi_{j}(t) \psi_{2^{n}}{ }^{n}(t) D_{k^{\prime}}(t) d t \\
& =\sum_{j=0}^{2^{n-1}} c_{2 n+j} \int_{2^{-\left[r^{r} n\right]}}^{1} \psi_{2 n+j}(t) \psi_{2 n}(t) D_{k^{\prime}}(t) d t \\
& =\sum_{j=0}^{2^{n}-1} c_{2 n+j} \int_{2^{-[r n]}}^{1} \psi_{j}(t) D_{k^{\prime}}(t) d t \quad\left(\text { since } \psi_{22^{n+j}}(t)=\psi_{2 n}(t) \psi_{j}(t)\right) \\
& =\sum_{p=0}^{2^{n-[r n]}} \sum_{j=p^{[}\left[^{[r n]}\right.}^{(p+1) 2^{[r n]}-1} c_{2 n+j}^{1} \int_{2^{-[r n]}}^{1} \psi_{j}(t) D_{k^{\prime}}(t) d t .
\end{aligned}
$$

Let $k^{\prime}=q 2^{[r n]}+s, 0 \leqq s<2^{[r n]}, 0 \leqq q<2^{n-[r n]}$. Since (see Fine $[1 ;(6,1)]$ )

$$
D_{k^{\prime}}(t)=D_{2[r n]}(t) D_{q}\left(2^{[r n]} t\right)+\psi_{q 2[r n]}(t) D_{s}(t)
$$

and $D_{2[}[r n](t)=0$ for $2^{-[r n]} \leqq t<1$, we get 


$$
\begin{aligned}
& T=\sum_{p=0}^{2^{-[r n]}} \sum_{j=p 2^{-1}[r n]}^{(p+1) 2^{[r n]}-1} c_{2 n+j} \int_{2^{-[r n]}}^{1} \psi_{j}(t) \psi_{q 2^{2}}[r n](t) D_{s}(t) d t \\
& =\sum_{p=0}^{2^{n-[r n]}-1} \sum_{j=0}^{2^{[r n]}-1} c_{2 n+p 2^{[r n]}}+j \int_{2^{-[r n]}}^{1} \psi_{p 2^{n+j}}(t) \psi_{q 2}{ }^{[r n]}(t) D_{s}(t) d t \\
& =\sum_{p=0}^{2^{n-[r n]}} \sum_{j=0}^{2_{2}^{[r n]}-1} c_{3 n+, y_{2}}^{[r n]}+\int_{-[r n]}^{1} \psi_{y 2 n}(t) \psi_{q_{2}[}^{[r n]}(t) \psi_{j}(t) D_{s}(t) d t .
\end{aligned}
$$

If $p \neq q, \psi_{p 2}^{[r n]}(t) \psi_{q_{2}}{ }^{[r n]}(t)$ reduces to a $\psi_{v}(t)$ where $v=u 2^{[r n]}, u>0$, so that it follows by (8) that

$$
\int_{2^{-[r n]}}^{1} \psi_{p 2^{[r n]}}(t) \psi_{q^{2}}{ }^{[r n]}(t) \psi_{j}(t) D_{s}(t) d t=0, \quad\left(\begin{array}{c}
0 \leqq j, s<2^{[r n]} \\
p \neq q
\end{array}\right) .
$$

Consequently

$$
\begin{aligned}
& T=\sum_{j=0}^{2^{r n]}-1} c_{2 n+q 2^{2}}^{[r n]^{2}}+\int_{2^{-[r n]}}^{1} \psi_{q 2^{2}}{ }^{[r n]}(t) \psi_{q 2^{2}}[r n](t) \psi_{j}(t) D_{s}(t) d t \\
& =\sum_{j=0}^{2^{[r n]}-1} c_{2 n+q 2^{[r n]}}+\int_{2^{-[r n]}}^{1} \psi_{j}(t) D_{s}(t) d t .
\end{aligned}
$$

Since it is known (Fine [1; Theorem IX (iii)]) that

$$
\int_{0}^{1}\left|D_{s}(t)\right| d t=O(\log s)
$$

it follows under condition (ii) that

$$
\begin{aligned}
|T| & \leqq \sum_{j=0}^{2^{[r n]}-1}\left|c_{\eta^{n n+q 2}}^{[r n]}+\jmath\right| \int_{0}^{1}\left|D_{s}(t)\right| d t \\
& =O\left(2^{-n \delta} \log s\right) 2^{[r n]} \\
& =O\left(n 2^{-[r n]}\right)=o(1),
\end{aligned}
$$

and this gives the desiered result.

We shall now prove the lemma. The proof is completely analogous to the one in the case of trigonometric Fourier series (see, for example, Zygmund [5; p. 91]), so that we shall give here a brief sketch.

Let the $n$-th partial sum of $h(x)$ be

$$
s_{n}(x ; h)=\sum_{k=0}^{n-1} b_{k} \psi_{k}(x)
$$

Then the uniform boundedness of the arithmetic means $\frac{1}{n} \sum_{k=1}^{n} s_{k}(x ; h)$ 
$=\sigma_{n}(x ; h)$ is easily verified by the relations

$$
\sigma_{n}(x ; h)=\int_{0}^{1} h(x+t) K_{n}(t) d t
$$

and (see [4; Lemma 9])

$$
\int_{0}^{1}\left|K_{n}(t)\right| d t \leqq 2
$$

where $K_{n}(t)$ is the Fejér kernel for the Walsh functions :

$$
K_{n}(t)=\frac{1}{n} \sum_{k=1}^{n} D_{k}(t)
$$

Since $h(x)$ is of bounded variation, $b_{n}=O\left(\frac{1}{n}\right)$ (Fine [1; Theorem VI]). From the relation

$$
s_{n}(x ; h)-\sigma_{n}(x ; h)=\frac{1}{n} \sum_{k=0}^{n-1} k b_{k} \psi_{k}(x),
$$

it is shown that the partial sum $s_{n}(x ; h)$ is uniformly bounded, and it converges to $h(x)$ at the continuity point $x$ of $h(x)$. Therefore, as $n \rightarrow \infty$ in the equality

we obtain the desired result.

$$
\int_{0}^{1} \chi(x) s_{n}(x ; h) d x=\sum_{k=0}^{n-1} a_{k} b_{k},
$$

\section{REFERENCES}

[1] N. J. Fine, On the Walsh functions, Trans. Amer. Math. Soc. vol. 65 (1949) 327414.

[2] G. H. HARdy and J. E. Littlewojd, Some new convergence criteria for Fourier series, Annali di Pisa, vol. 34 (1935).

[3] R. E. A.C. PALEY, A remarkable series of orthogonal functions, Proc. London Math. Soc. vol. 34 (1935) 247-279.

[4] S. YANO, On Walsh-Fourier series, Tôhoku Math. Jour. 3 (1951) 223-242.

[5] A. ZYGMUND, Trigonometrical series, Walsow (1935)

Mathematical Institute, TOKyo Metropolitan University. 